\title{
Deep Vein Thrombosis of the Left Leg: A Case of May-Thurner Syndrome
}

\author{
Jiten Desai ${ }^{1}$, Zalak Desai ${ }^{1}$, Jay Shah ${ }^{2}$, Ofek Hai $^{1}$, Andrea Mignatti ${ }^{1}$, Roman Zeltser ${ }^{1}$, Amgad Makaryus $^{1}$ \\ ${ }^{1}$ Nassau University Medical Center, East Meadow, NY, USA \\ ${ }^{2}$ Saint Vincent Mercy Medical Center, Toledo, OH, USA
}

Received: $19 / 12 / 2017$

Accepted: 03/01/2018

Published: 08/02/2018

How to cite this article: Desai J, Desai Z, Shah J, Hai O, Mignatti A, Zeltser R, Makaryus A. Deep vein thrombosis of the left leg: a case of May-Thurner syndrome. EJCRIM 2018;5: doi:10.12890/2018_000829.

Conflicts of Interests: The Authors declare that there are no competing interests.

Additional Authors: Charudatta Wankhade, Paresh Sojitra, Andrew Cantos, Salman Shah, Nassau University Medical Center, East Meadow, NY, USA

This article is licensed under a Commons Attribution Non-Commercial 4.0 License

\section{ABSTRACT}

A 56-year-old woman presented with gradually worsening shortness of breath associated with dull left leg pain over 5 days. She denied any recent travel, recent surgeries or immobilization.

CT pulmonary angiography and CT venography revealed multiple bilateral pulmonary emboli and extensive left pelvic and left lower extremity deep vein thromboses. Contrast-enhanced CT showed that the right common iliac artery crossed the left common iliac vein and compressed it externally, indicative of May-Thurner syndrome. Catheter-directed thrombolysis of the left lower extremity was performed and heparin infusion was started. The patient also underwent left iliac vein balloon angioplasty with stenting and infra-renal inferior vena cava filter placement via the jugular approach to prevent further embolization.

\section{LEARNING POINTS}

- May-Thurner syndrome (MTS) should be suspected in patients in their second to fourth decade of life presenting with unprovoked deep venous thrombosis of the left leg.

- An iliac venogram is the diagnostic test of choice for MTS.

- MTS is treated only when it is symptomatic. The goal of treatment is to remove the clot to prevent post-thrombotic syndrome and to repair the anatomical defect.

- A stent was successfully deployed in the iliac vein of our patient and restoration of blood flow resulted in a dramatic improvement in the patient's symptoms.

\section{KEYWORDS}

May-Thurner syndrome, deep venous thrombosis, left common iliac vein, catheter-directed venous thrombolysis, balloon angioplasty, stent

\section{INTRODUCTION}

In 1851, Virchow observed that iliofemoral deep venous thrombosis (DVT) was five times more common in the left leg compared with the right. In 1943, Ehrich and Krumbhaar performed anatomic dissections in 412 cadavers and found obstructive lesions in $23.8 \%$ of the left common iliac veins. In 1957, May and Thurner provided an explanation for this phenomenon when they discovered an anatomical variation of the left common iliac vein. They found that the left common iliac vein had vascular thickening at the point where it was crossed and compressed against the fifth lumbar vertebra by the overlying right common iliac artery ${ }^{[1]}$. They called this lesion 'a venous spur' and 
postulated that chronic pulsation of the overlying iliac artery is responsible for formation of this spur and that it is the spur that leads to venous obstruction. This anatomic variant later became widely known as May-Thurner syndrome (MTS).

\section{CASE REPORT}

A 56-year-old African-American woman with a past medical history of rheumatoid arthritis presented with gradually worsening shortness of breath associated with dull left leg pain over 5 days. She denied any recent travel, recent surgeries or immobilization. Her home medications included prednisone and golimumab. She had smoked half a pack of cigarettes per day since the age of 20 , and denied alcohol usage.

The patient also reported swelling of the left lower extremity multiple times in the past for which she had never sought any medical attention. On physical examination, she was afebrile with a warm left lower extremity that was swollen and erythematous from the ankle to mid-thigh. Distal pulses, motor strength and sensations were intact. Laboratory tests showed the patient had mild anaemia. Prothrombin time was $13.6 \mathrm{sec}$, INR was 1.1 and partial thromboplastin time (PTT) was $30.5 \mathrm{sec}$. CT pulmonary angiography and CT venography revealed multiple bilateral pulmonary emboli and extensive left pelvic and left lower extremity DVT. Contrast-enhanced CT at the level of the pelvis also showed the right common iliac artery crossed the left common iliac vein and compressed it externally, indicative of May-Thurner syndrome (MTS) (Figs. 1 and 2). The patient was given one dose of enoxaparin in the emergency department. Catheter-directed thrombolysis of the left lower extremity extending from the inferior vena cava (IVC) to the left popliteal vein was performed with alteplase. Heparin infusion was started with a PTT goal of 40-60 sec. The patient also underwent left iliac vein balloon angioplasty with stenting and infra-renal IVC filter placement via the jugular approach to prevent further embolization (Figs. 3 and 4). All other causes of thrombophilia were ruled out and the diagnosis of MTS was made. Heparin therapy was bridged with warfarin therapy with an INR goal of 2-3.

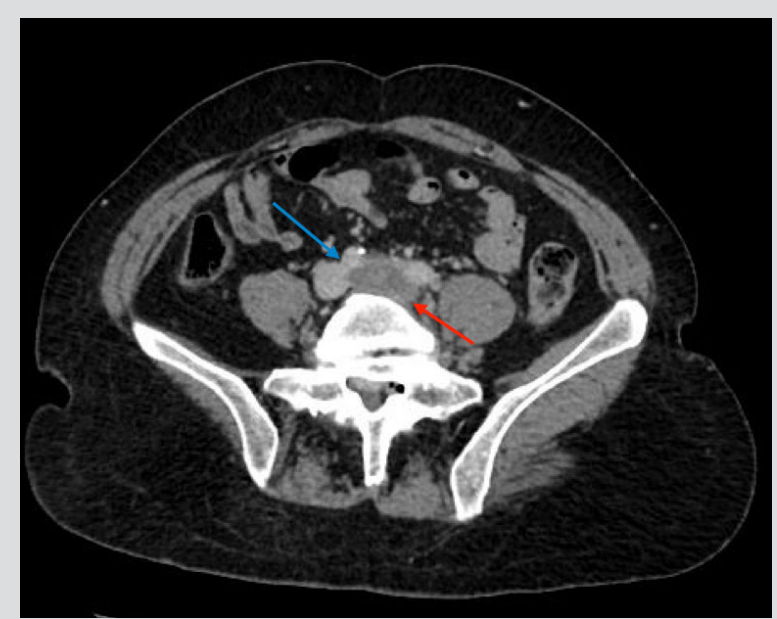

Figure 1. Axial contrast-enhanced CT at the level of the pelvis demonstrates extensive thrombus in the left common iliac vein (red arrow) extending to the distal inferior vena cava. The right common iliac artery (blue arrow) is seen crossing just superior to the left common iliac vein, indicative of May-Thurner syndrome. Extensive thrombus is seen extending as far as the left popliteal vein (not imaged)

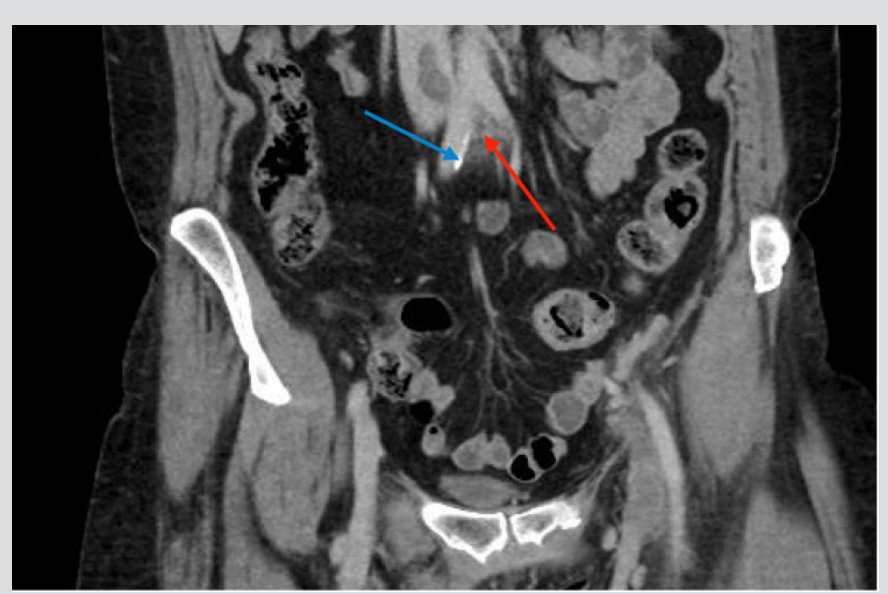

Figure 2. Coronal contrast-enhanced CT demonstrates the right common iliac artery (blue arrow) crossing over the left common iliac vein (red arrow) in a different plane

\section{DISCUSSION}

A history of persistent left lower extremity swelling with or without DVT in a woman between the second and fourth decades of life, without an obvious cause, is highly suggestive of MTS. Patients with a known diagnosis of MTS should always be evaluated for thrombophilia, as Kolbel et al. found that $67 \%$ of patients with chronic iliac vein occlusion or MTS have some form of thrombophilia ${ }^{[2]}$. An iliac venogram is the diagnostic test of choice. Contrast material-enhanced CT scans at the level of the distal abdominal aorta can also demonstrate the extrinsic compression caused by the right common iliac artery. Doppler ultrasound will detect the DVT lesion but is unable to visualize iliac vein compression or spurs ${ }^{[3]}$. Other causes of iliac vein compression, like pelvic mass, trauma and prior surgeries, should be ruled out before diagnosing MTS. Our patient had a classic form of MTS and no evidence of thrombophilia.

MTS is treated only when it is symptomatic. If a DVT occurs, the goal of treatment is to remove the clot with pharmaco-mechanical 


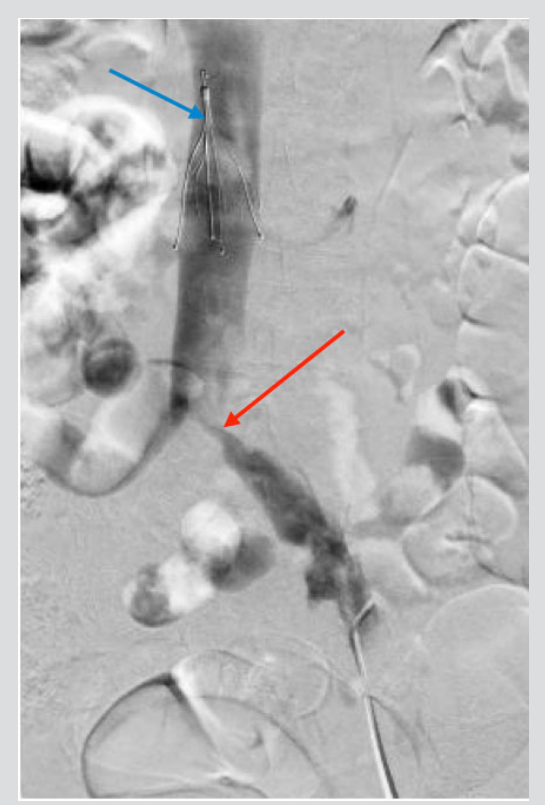

Figure 3. Left common iliac vein injection reveals a severely stenotic lesion at the level of the iliac vein bifurcation (red arrow). An inferior vena cava filter (blue arrow) is noted and was placed prior to popliteal vein access in an attempt to prevent distal emboli

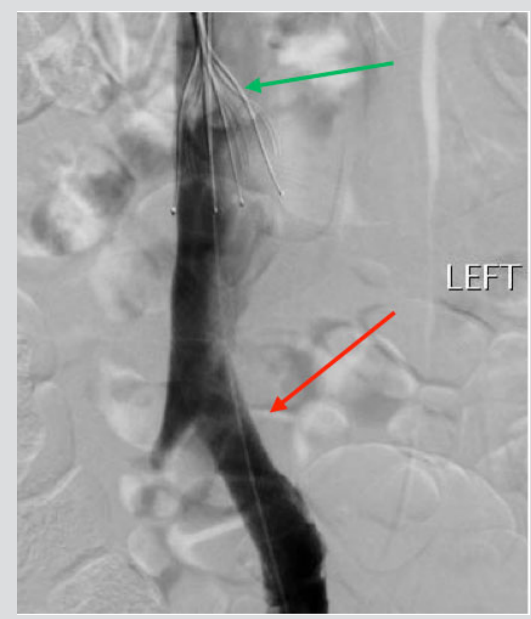

Figure 4. Left common iliac vein injection after angioplasty and stenting shows significant improvement in the left common iliac vein stenosis (red arrow). An incidentally noted thrombus is seen beneath the inferior vena cava filter (green arrow)

thrombolysis to prevent post-thrombotic syndrome (PTS) and to repair the anatomical defect with the use of stents and balloon venoplasty ${ }^{[4]}$. Recent results from a catheter-directed venous thrombolysis (CDT) study demonstrated a significant reduction in the incidence of PTS in the CDT treatment arm compared with the traditional anticoagulation group at 2 years $(41 \% \text { vs } 56 \%, p=0.047)^{[5]}$.

The manifestations of PTS include leg pain, swelling, claudication, skin hyperpigmentation, venous varicosities and rarely venous stasis ulcers. Following placement of stents, DVT needs to be treated with oral anticoagulants for at least 6 months.

The first known report of treatment of MTS solely by endovascular means was in 1995 by Berger et al., who successfully placed a venous stent to relieve iliac compression ${ }^{[6]}$. Some authors suggest that MTS-related DVT patients with a high clot burden should also undergo IVC filter placement ${ }^{[7]}$. Multiple surgical treatment options considered in the past include vein-patch angioplasty with excision of the intraluminal bands, division of the right common iliac artery and relocation behind the left common iliac vein or inferior vena cava, and contralateral saphenous vein graft bypass to the ipsilateral common femoral vein with creation of a temporary arteriovenous fistula ${ }^{[3]}$. The unique aspect of this case was that a stent was successfully deployed in the iliac vein (minimally invasive endovascular treatment) and that restoration of flow resulted in a dramatic improvement in the patient's symptoms.

\section{REFERENCES}

1. May R, Thurner J. The cause of the predominately sinistral occurrence of thrombosis of the pelvic veins. Angiology 1957;8:419-427.

2. Kolbel T, Lindh M, Akesson M, Wasselius J, Gottsater A, Ivancev K. Chronic iliac vein occlusion: midterm results of endovascular recanalization. J Endovasc Ther 2009;16:483491.

3. Patel NH, Stookey KR, Ketcham DB, Cragg AH. Endovascular management of acute extensive iliofemoral deep venous thrombosis caused by May-Thurner syndrome. J Vasc Interv Radiol 2000;11:1297-1302.

4. Raffini L, Raybagkar D, Cahill AM, Kaye R, Blumenstein M, Manno C. May-Thurner syndrome (iliac vein compression) and thrombosis in adolescents. Pediatr Blood Cancer 2006;47:834-838

5. Enden T, Haig Y, Kløw NE, Slagsvold CE, Sandvik L, Ghanima W, et al. Long-term outcome after additional catheter-directed thrombolysis versus standard treatment for acute iliofemoral deep vein thrombosis (the CaVenT study): a randomised controlled trial. Lancet 2012;379:31-38.

6. Berger A, Jaffe JW, York TN. Iliac compression syndrome treated with stent placement. J Vasc Surg 1995;21:510-514.

7. Moudgill N, Hager E, Gonsalves C, Larson R, Lombardi J, Dimuzio P. May-Thurner syndrome: case report and review of the literature involving modern endovascular therapy. Vascular 2009;17:330-335. 